\title{
A PROOF OF A FUNCTIONAL EQUATION RELATED TO THE THEORY OF PARTITIONS
}

\section{SHÔ ISEKI}

Introduction. The purpose of this note is to give a simple alternative proof for the following functional equation:

$$
\begin{array}{r}
\sum_{l=0}^{\infty}\{\lambda((l+\alpha) z-i \beta)+\lambda((l+1-\alpha) z+i \beta)\}+\pi z\left(\alpha^{2}-\alpha+1 / 6\right) \\
=\sum_{l=0}^{\infty}\left\{\lambda\left((l+\beta) z^{-1}+i \alpha\right)+\lambda\left((l+1-\beta) z^{-1}-i \alpha\right)\right\} \\
+\pi z^{-1}\left(\beta^{2}-\beta+1 / 6\right)+2 \pi i(\alpha-1 / 2)(\beta-1 / 2),
\end{array}
$$

where $\lambda(t)=-\log \left(1-e^{-2 \pi t}\right)$ (the principal value), $0 \leqq \alpha \leqq 1,0<\beta<1$ (or $0<\alpha<1,0 \leqq \beta \leqq 1$ ), and $\Re(z)>0$. This formula was first proved in [2], and its applications to the theory of partitions have also been investigated (see $[2 ; 3]$ ).

The method we employ in this paper is essentially that of Rademacher [4]. Although our method is still based on the Mellin transform technique and the theory of the Hurwitz zeta-functions, we have made a thorough revision of Rademacher's original method, and thus a very short and direct proof of the functional equation can be given.

It may be noted that if we define a function $\Lambda(z, \alpha, \beta)$ by

$$
\begin{aligned}
\Lambda(z, \alpha, \beta)= & \sum_{l=0}^{\infty}\{\lambda((l+\alpha) z-i \beta)+\lambda((l+1-\alpha) z+i \beta)\} \\
& +\pi z\left(\alpha^{2}-\alpha+1 / 6\right)-\pi i(\alpha-1 / 2)(\beta-1 / 2),
\end{aligned}
$$

then (1) is written in the form

$$
\Lambda(z, \alpha, \beta)=\Lambda\left(z^{-1}, 1-\beta, \alpha\right) .
$$

On the other hand, it is clear from the definition that

$$
\Lambda(z, \alpha, \beta)=\Lambda(z, 1-\alpha, 1-\beta) .
$$

Proof of the functional equation. To prove (1), we first assume that $0<\alpha<1,0<\beta<1, \Re(z)>0$. Using the expansion

$$
\lambda(x)=-\log \left(1-e^{-2 \pi x}\right)=\sum_{n=1}^{\infty} n^{-1} e^{-2 \pi x n}
$$

and applying the Mellin formula

Received by the editors May 13, 1960 and, in revised form, June 21, 1960. 


$$
e^{-y}=(2 \pi i)^{-1} \int_{c-i \infty}^{c+i \infty} \Gamma(s) y^{-s} d s \quad(\Re(y)>0)
$$

with $c=3 / 2$, it follows that

$$
\begin{aligned}
& \sum_{l=0}^{\infty} \lambda((l+\alpha) z-i \beta)=\sum_{l=0}^{\infty} \sum_{n=1}^{\infty} \frac{1}{n} e^{-2 \pi((l+\alpha) z-i \beta) n} \\
& =\sum_{l=0}^{\infty} \sum_{n=1}^{\infty} \frac{e^{2 \pi i \beta n}}{2 \pi i n} \int_{3 / 2-i \infty}^{3 / 2+i \infty} \Gamma(s)\{2 \pi(l+\alpha) z n\}^{-s} d s \\
& =\frac{1}{2 \pi i} \int_{3 / 2-i \infty}^{3 / 2+i \infty} \frac{\Gamma(s)}{(2 \pi z)^{s}}\left(\sum_{l=0}^{\infty} \frac{1}{(l+\alpha)^{s}}\right)\left(\sum_{n=1}^{\infty} \frac{e^{2 \pi i \beta n}}{n^{1+s}}\right) d s .
\end{aligned}
$$

Therefore

(2) $\sum_{l=0}^{\infty} \lambda((l+\alpha) z-i \beta)=\frac{1}{2 \pi i} \int_{3 / 2-i \infty}^{3 / 2+i \infty} \frac{\Gamma(s)}{(2 \pi z)^{s}} \zeta(s, \alpha) \zeta_{\beta}(1+s) d s$,

where $\zeta(s, \alpha)=\sum_{l=0}^{\infty}(l+\alpha)^{-s}$ is the Hurwitz zeta-function, and $\zeta_{\beta}(s)$ $=\sum_{n=1}^{\infty} \dot{n}^{-s} e^{2 \pi i \beta n}(\Re(s)>0)$.

Now the function $\zeta_{a}(s)(0<a<1)$ can be expressed in terms of Hurwitz zeta-functions, i.e.,

$$
\begin{aligned}
\zeta_{a}(s)=\Gamma(1-s)(2 \pi)^{s-1}\left\{e^{\pi i(1-s) / 2} \zeta(1-s, a)\right. \\
\left.+e^{\pi i(s-1) / 2} \zeta(1-s, 1-a)\right\} .
\end{aligned}
$$

This relation is an easy consequence of the famous formula:

$$
\begin{aligned}
& \zeta(s, a)=\frac{2 \Gamma(1-s)}{(2 \pi)^{1-s}}\left\{\sin (\pi s / 2) \sum_{n=1}^{\infty} \frac{\cos (2 \pi a n)}{n^{1-s}}\right. \\
&\left.\quad+\cos (\pi s / 2) \sum_{n=1}^{\infty} \frac{\sin (2 \pi a n)}{n^{1-s}}\right\} \quad(\Re(s)<0) .
\end{aligned}
$$

From (3) we see that $\zeta_{a}(s)$ is an integral function of $s$ (cf. Apostol $[1])$.

Returning to (2), we obtain further that

$$
\begin{aligned}
\sum_{l=0}^{\infty}\{\lambda((l+\alpha) z-i \beta)+\lambda((l+1-\alpha) z+i \beta)\} & \\
= & \frac{1}{2 \pi i} \int_{3 / 2-i \infty}^{3 / 2+i \infty} z^{-s} F(s ; \alpha, \beta) d s
\end{aligned}
$$

with 
(5) $\quad F(s ; \alpha, \beta)=\Gamma(s)(2 \pi)^{-\cdot}\left\{\zeta(s, \alpha) \zeta_{\theta}(1+s)+\zeta(s, 1-\alpha) \zeta_{1-\theta}(1+s)\right\}$.

The function $F(s ; \alpha, \beta)$ satisfies the equation

$$
F(s ; \alpha, \beta)=F(-s ; 1-\beta, \alpha),
$$

since we have, by (5) and (3),

$$
\begin{aligned}
& F(s ; \alpha, \beta) \\
& (7) \quad \Gamma(s) \Gamma(-s)\left[e^{-\pi i s / 2}\{\zeta(s, \alpha) \zeta(-s, \beta)+\zeta(s, 1-\alpha) \zeta(-s, 1-\beta)\}\right. \\
& \left.\quad+e^{\pi i s / 2}\{\zeta(s, \alpha) \zeta(-s, 1-\beta)+\zeta(s, 1-\alpha) \zeta(-s, \beta)\}\right],
\end{aligned}
$$

which is unchanged when $s, \alpha, \beta$ are replaced by $-s, 1-\beta, \alpha$ respectively.

Now in the integral on the right of (4), we move the path of integration from $\Re(s)=3 / 2$ to $\Re(s)=-3 / 2$, and obtain

$$
\begin{aligned}
\frac{1}{2 \pi i} \int_{3 / 2-i \infty}^{3 / 2+i \infty} z^{-\cdot} F(s ; \alpha, \beta) d s & \\
& =\frac{1}{2 \pi i} \int_{-3 / 2-i \infty}^{-8 / 2+i \infty} \tau^{-\cdot} F(s ; \alpha, \beta) d s+R_{1}+R_{0}+R_{-1},
\end{aligned}
$$

where $R_{1}, R_{0}, R_{-1}$ are the corresponding residues of the integrand at its simple poles $s=1,0,-1$ respectively. The displacement of the path of integration may easily be justified by considering the order of magnitude of the integrand. Actually, we have, writing $\Im(s)=t$,

$$
\begin{array}{rlr}
z^{\prime} & =O\left(e^{(\pi / 2-\delta)|t|}\right) & \text { for }|\arg z| \leqq \pi / 2-\delta<\pi / 2, \\
\Gamma(s) \Gamma(-s) & =-\pi(s \sin \pi s)^{-1}=O\left(|t|^{-1} e^{-\pi|t|}\right), \\
\zeta(s, a) & =O(|t| c) & \text { (see [5, p. 276]), }
\end{array}
$$

and hence, by (7),

$$
z^{-s} F(s ; \alpha, \beta)=O\left(|t|^{2 c-1} e^{-\delta|t|}\right) .
$$

The values of the residues are calculated as follows:

$$
\begin{aligned}
R_{1} & =(2 \pi z)^{-1}\left\{\zeta_{\beta}(2)+\zeta_{1-\beta}(2)\right\} \\
& =(2 \pi z)^{-1} \sum_{n=1}^{\infty} n^{-2}\left(e^{2 \pi i \beta n}+e^{-2 \pi i \beta n}\right) \\
& =(\pi z)^{-1} \sum_{n=1}^{\infty} n^{-2} \cos (2 \pi \beta n)=\pi z^{-1}\left(\beta^{2}-\beta+1 / 6\right),
\end{aligned}
$$




$$
\begin{aligned}
R_{0} & =\zeta(0, \alpha) \zeta_{\beta}(1)+\zeta(0,1-\alpha) \zeta_{1-\beta}(1) \\
& =(1 / 2-\alpha) \sum_{n=1}^{\infty} n^{-1}\left(e^{2 \pi i \beta n}-e^{-2 \pi i \beta n}\right) \\
& =2 i(1 / 2-\alpha) \sum_{n=1}^{\infty} n^{-1} \sin (2 \pi \beta n) \\
& =2 \pi i(1 / 2-\alpha)(1 / 2-\beta),
\end{aligned}
$$

and, using (6),

$$
R_{1}=-\pi z\left(\alpha^{2}-\alpha+1 / 6\right) .
$$

Inserting these values and the relation (6) into the right member of (8), changing the variable $s$ to $-s$ and using (4), it is found that the required equation (1) holds for $0<\alpha<1,0<\beta<1$, and $\Re(z)>0$. The validity of (1) for the end-points of the interval of $\alpha$ or $\beta$ may then be established by means of letting $\alpha \rightarrow+0,1-0$ or $\beta \rightarrow+0,1-0$ in (1). This completes the proof of the functional equation.

\section{BIBLIOGRAPHY}

1. T. M. Apostol, On the Lerch zeta function, Pacific J. Math. vol. 1 (1951) pp. 161-167.

2. S. Iseki, The transformation formula for the Dedekind modular function and related functional equations, Duke Math. J. vol. 24 (1957) pp. 653-662.

3. - A partition function with some congruence condition, Amer. J. Math. vol. 81 (1959) pp. 939-961.

4. H. Rademacher, Zur Theorie der Modulfunktionen, J. Reine Angew. Math. vol. 167 (1932) pp. 312-336.

5. E. T. Whittaker and G. N. Watson, A course of modern analysis, Cambridge, University Press, 1945.

Defense Academy, Yokosuka, Japan 